\title{
The Electrical Resistivity of the Double Exchange Interaction Systems
}

\author{
Chong Der Hu \\ Department of Physics, National Taiwan University, Taipei, Taiwan, R.O.C.
}

(Received July 12, 2000)

\begin{abstract}
We used the Boltzmann equation to calculate the electrical resistivity of the double exchange interaction systems. Starting from the Hamiltonian developed by [Kubo and Ohata's Hamiltonian J. Phys. Soc. Jpn. 33 (1972) 21], we calculated the resistivity due to the spin wave scattering. It was found that below $T_{\mathrm{C}}$, the behavior of resistivity was quite complicated and most importantly, its magnitude is small compared to experimental results.
\end{abstract}

KEYWORDS: colossal magnetoreisistance, manganite, electrical resistivity

\section{§1. Introduction}

In the past several years, a lot of effort has been devoted to the study of the perovskite manganites. They showed huge negative magnetoresistance below Curie temperature which is called 'colossal magnetoresistance' (CMR). ${ }^{1,2)}$ The representative compound is $\mathrm{La}_{1-x} \mathrm{~A}_{x} \mathrm{MnO}_{3}$ where $A$ represents a divalent element. Owing to different doping concentration $x$, lattice distortion and temperature effect, these compounds exhibit many phases under different conditions, such as antiferromagnetic insulator, ferromagnetic insulator, ferromagnetic metal, charge ordering and paramagnetic insulator. The most prominent feature is the coupling between magnetic properties and transport properties. This can be understood in terms of the double exchange (DE) model. ${ }^{3)}$ An electrons can hop from an intermediate oxygen ion to an $\mathrm{Mn}^{+4}$ ion while another hops from a neighboring $\mathrm{Mn}^{+3}$ ion to the oxygen ion. Due to the on-site Coulomb repulsion, the electron spin must be parallel to the spins of Mn ions (Hund's rules) and thus creating magnetic coupling. As a result, if the local spins of $\mathrm{Mn}^{+4}$ and $\mathrm{Mn}^{+3}$ are parallel, then the electrons can hop easily and have extended wave functions and lower energies. Thus, ferromagnetic metallic state is favorable at low temperature. As temperature rises, the directions of local spins become random and the system goes into paramagnetic insulating state. DE had been further developed by Anderson and Hasegawa ${ }^{4)}$ and De Gennes. ${ }^{5)}$ Next major progress was made by Kubo and Ohata ${ }^{6)}$ (to be referred as KO). Since the 'Hund's rule' coupling is very large and not solvable. They used projection operators to project out the lowest-energy states according to Hund's rule and do the calculation only in these states, thereby perserve the most important physics in low energy. Furukawa ${ }^{7)}$ used another approach: the infinite dimension Kondo lattice model to gain some insight of the double exchange interaction. Shen et al. ${ }^{8)}$ proposed that Andersion localization was responsible for various properties of manganites. Recent research showed that manganites have richer physics, namely the Jahn-Teller effect ${ }^{9)}$ and charge and orbital ordering. ${ }^{10)}$ In this paper we focus our attention to the resistivity in the ferromagnetic metallic phase, i.e. doping concentration $0.2<x<0.5$ in a pure double exchange interaction system.

Experiments $^{11}$ ) showed that below $T_{\mathrm{C}}$ the resistivity is proportional to $T^{2}$. KO calculated the resistivity of the double exchange model and found that the resistivity is proportional to $T^{9 / 2}$. However, the calculation is only applicable in very low temperature. Hence, it is not appropriate to compare KO's result with experiments. At finite temperature many factors can affect resistivity. First of all there is spin-flip scattering. Energy conservation renders this process insignificant in low temperature but it is not negligible in higher temperature range. Furthermore, the energy bands are affected by temperature. The effect of band structure is three-fold. The effective masses of holes vary with magnetization and thus, with temperature. Also, as the system approaches the Curie temperature, spin-up band and spin-down band move closer to each other and the scattering rate is enhanced. Thirdly, the numbers of spin-up holes and spindown holes vary with temperature. Lastly, the spin wave stiffness and the coupling between holes and spin waves vary with magnetization and temperature. Therefore, it is worthwhile to perform a detailed calculation of resistivity at finite temperature. We introduced the Hamiltonian is in $\S 2$ and gave the details of the calculation in $\S 3$. Results and conclusion are in $\S 4$.

\section{§2. Hamiltonian}

In the CMR manganites, the energy spacing of the states of a $\mathrm{Mn}^{+3}$ ion is large compared to the band width. ${ }^{12)}$ Taking advantage of this fact, KO proposed the following Hamiltonian to project out the states which have the lowest energies according to the Hund's rules:

$$
H=-t \sum_{i, j, \alpha, \beta} c_{i \alpha}^{\dagger}\left(P_{i} P_{j}\right)_{\alpha \beta} c_{j \beta},
$$

where $i$ and $j$ are nearest neighbors and

$$
P_{i}=\frac{S-\boldsymbol{S}_{i} \cdot \boldsymbol{\sigma}}{2 S+1} .
$$

Within the Hamiltonian, the manganese ions can be $\mathrm{Mn}^{+4}$, which has spin $3 / 2$, or $\mathrm{Mn}^{+3}$ which has spin $S=2$. Equation (1) describes the hopping of the holes 
between $\mathrm{Mn}^{+3}$ and $\mathrm{Mn}^{+4}$ sites. The projection operators impose the restriction on holes. Hoppings can only occur at those sites where the local spins are antiparallel to those of holes. At finite temperatures, the spins of manganese ions are not always aligned, hence, there exist spin-up and spin-down holes. In the process of hopping, the spins of holes can be flipped though the total spin is conserved.

The Hamiltonian can be expanded as

$$
\begin{aligned}
H= & -t \sum_{i, j \alpha, \beta} c_{i \alpha}^{\dagger}\left[S^{2}+\boldsymbol{S}_{i} \cdot \boldsymbol{S}_{j}-\left(S S_{i z}+S S_{j z}-\frac{1}{2} S_{i-} S_{j+}+\frac{1}{2} S_{i+} S_{j-}\right) \sigma_{z}\right. \\
& \left.-\left(S S_{i-}+S S_{j-}-S_{i z} S_{j-}+S_{i-} S_{j z}\right) \frac{\sigma_{+}}{2}-\left(S S_{i+}+S S_{j+}+S_{i z} S_{j+}-S_{i+} S_{j z}\right) \frac{\sigma_{-}}{2}\right]_{\alpha \beta} c_{j \beta} .
\end{aligned}
$$

Above Hamiltonian can be devided into a mean field part and a spin-fluctuation part. In the mean field part we are going to have hole bands and spin wave energy. The hole bands are magnetization-dependent, and hence, temperature-dependent. The holes of majority spin have a lower energy band and smaller effective mass. The local spins interact with each other through the holes. Thus, we expect there are spin waves and the dispersion relationis is related to the spin and charge density polarizations of holes. In the mean field part we included the dressed spin wave energy.

In the process of hopping, a hole will also experience the scattering by spin fluctuations. There are two kinds of fluctuations: dressed spin waves and the fluctuations of $S_{z}$. Only the former is important for resistivity. The reason will be given later. The fluctuation part can be treated perturbatively for temperature below $T_{\mathrm{C}}$ for the following reasons. Higher order terms involve more hoppings. A hole can have an extensive wave function so that its kinetic energy can be reduced. However, it will sample spin-fluctuations on many sites. These fluctuations have random phases and in general have destructive interference. On the other hand, a more localized hole is more strongly perturbed by spin fluctuations, but it is less important because of its higher kinetic energy. Therefore, the contribution of the higher order terms to physical properties will be small. A detailed analysis was given in a previous paper. ${ }^{13)}$ In general, the perturbation of spin fluctuation has a small factor $1 /(2 S+1)$ for temperature below $T_{\mathrm{C}}$. The hole density also plays a role. As shown by $\mathrm{KO}$, the spin wave frequency is proportional to the hole density. The hole-hole interaction, however, will not have significant contribution to resistivity unless orbital ordering is important. because in the metallic region the interaction will be screened. Our calculation will not be accurate when $x$ is close to 0.5 , where the orbital ordering effect is important. Therefore, we restrict our calculation in the region $0.2<x<0.5$. Now we have the effective Hamiltonian as

$$
H=H_{m f}+H_{f l},
$$

where

$$
\begin{aligned}
H_{m f}= & \frac{-t}{N(2 S+1)^{2}} \sum_{i, j, \boldsymbol{k}, \sigma} \mathrm{e}^{\mathrm{i} \boldsymbol{k} \cdot \boldsymbol{R}_{i j}}\left(S-\sigma\left\langle S_{z}\right\rangle\right)^{2} c_{\boldsymbol{k}, \sigma}^{\dagger} c_{\boldsymbol{k}, \sigma} \\
& +\frac{1}{S^{2}-\left\langle S_{z}^{2}\right\rangle+S-\left\langle S_{z}\right\rangle} \sum_{\boldsymbol{q}} \omega_{\boldsymbol{q}} S_{\boldsymbol{q}-} S_{\boldsymbol{q}+}
\end{aligned}
$$

and

$$
H_{f l}=H_{f l 1}+H_{f l 2} .
$$

with

$$
\begin{aligned}
H_{f l 1}= & \frac{-t}{N(2 S+1)^{2}} \sum_{i, j, \boldsymbol{k}, \boldsymbol{q}} \mathrm{e}^{\mathrm{i} \boldsymbol{k} \cdot \boldsymbol{R}_{i j}+\mathrm{i} \boldsymbol{q} \cdot \boldsymbol{R}_{i}}\left[c_{\boldsymbol{k}-\boldsymbol{q},+}^{\dagger} c_{\boldsymbol{k},-}\left(S_{i z} S_{i-}-S_{i-} S_{j z}-S S_{i-}-S S_{j-}\right)\right. \\
& \left.+c_{\boldsymbol{k}-\boldsymbol{q},-}^{\dagger} c_{\boldsymbol{k},+}\left(-S S_{i+}-S S_{j+}+S_{i+} S_{j z}-S_{i z} S_{j+}\right)\right] \\
H_{f l 2}= & \frac{-t}{N(2 S+1)^{2}} \sum_{i, j, \boldsymbol{k}, \boldsymbol{q}, \sigma} c_{\boldsymbol{k}-\boldsymbol{q}, \sigma}^{\dagger} c_{\boldsymbol{k}, \sigma}\left(S_{i-} S_{j+}+S_{i+} S_{j-}\right)
\end{aligned}
$$

Here, $\langle X\rangle$ is the thermal average of $X$, and $\sigma= \pm 1$ representing spin-up and spin down holes. In view of the mean field part, we found that as temperature rises, $\left\langle S_{z}\right\rangle$ becomes smaller and two bands move closer to each other.The energy of an hole with spin polarization $\sigma$ is

$$
\varepsilon_{\boldsymbol{k}, \sigma}=-\left(S-\sigma\left\langle S_{z}\right\rangle\right)^{2} \varepsilon_{\boldsymbol{k}} .
$$

where

$$
\varepsilon_{\boldsymbol{k}}=\frac{t}{(2 S+1)^{2}} \sum_{n . n .} \mathrm{e}^{\mathrm{i} \boldsymbol{k} \cdot \boldsymbol{R}} .
$$

with $\sum_{n . n}$. sums over nearest neighbors. To reduce the amount of computation, we used a free hole band in place of that in eq. (9).

$$
\varepsilon_{\boldsymbol{k}}=-\frac{t\left(b k^{2}-z\right)}{(2 S+1)^{2}}
$$

where $\boldsymbol{k}$ is the crystal momentum in unit of $\pi / a$. If we expand $\varepsilon_{\boldsymbol{k}}$ in eq. (10) in powers of $\boldsymbol{k}$ we will have $b=\pi^{2}$. Since we are interested in the situations of doping less than 0.5 , only the lower-half of the band is of importance eq. (11) with $b=6.5$ simulated the lowerhalf band of eq. (10) quite well. The dispersion relation of spin waves were calculated. ${ }^{14)}$ To reduce the amount of computation, we used the empirical formulus which gives reasonably good fit to experimental data. ${ }^{15)}$ 


$$
\omega_{\boldsymbol{q}}=D_{s} q^{2} \simeq S x \varepsilon_{\boldsymbol{k},-}\left(1-\frac{T}{T_{\mathrm{C}}}\right)^{1 / 3} q^{2} .
$$

Equation (12) reduced to the low-temperature limit given by $\mathrm{KO}$. In the calculation of resistivity, we will consider the scattering of holes by dressed spin waves.

The origins of the terms in eqs. (7) and (8) can be traced back to corresponding terms in eq. (3). Readers will notice that in $H_{f l}$ there is no coupling involving only $S_{z}$. Having extracted the mean field part from the coupling with $S_{z}$ we can neglect the fluctuations for our purpose. The expectation of the first order of fluctuations always vanishes. The second order of fluctuations simply gives an effective hole-hole interaction. Its contribution to resistivity involves Umklapp processes which are always very small.

\section{$\S 3 . \quad$ Boltzmann Equation}

The first order scattering from $H_{f l 1}$ is a spin-flip process for holes while that from $H_{f l 2}$ and the second order in $H_{f l 1}$ are spin-conserving processes. We shall take into account above mentioned scattering into the calculation of the resistivity. KO did not include the spin-flip process because their calculation was in the low temperature region. The scattering rate of a hole in the state $\boldsymbol{k}, \sigma=-1$ in a spin-flip process is

$$
\frac{2 \pi}{N \hbar} \sum_{\boldsymbol{q}} \delta\left(\varepsilon_{\boldsymbol{k},-}-\varepsilon_{\boldsymbol{k}-\boldsymbol{q},+}-\omega_{\boldsymbol{q}}\right)\left\langle S_{\boldsymbol{q}+} S_{\boldsymbol{q}-}\right\rangle\left|M_{\boldsymbol{k}, \boldsymbol{k}-\boldsymbol{q},-}\right|^{2}
$$

where

$$
M_{\boldsymbol{k}, \boldsymbol{k}-\boldsymbol{q}, \sigma}=\left[\left(S-\sigma\left\langle S_{z}\right\rangle\right) \varepsilon_{\boldsymbol{k}}+\left(S+\sigma\left\langle S_{z}\right\rangle\right) \varepsilon_{\boldsymbol{k}-\boldsymbol{q}}\right] .
$$

We calculate the matrix element of spin operators with the following relation:

$$
\begin{aligned}
\left\langle S_{+} S_{-}\right\rangle & =2\left\langle S_{z}\right\rangle+\left\langle S_{-} S_{+}\right\rangle \\
& =2\left\langle S_{z}\right\rangle+\mathrm{e}^{-\beta \omega}\left\langle S_{+} S_{-}\right\rangle \\
& =\frac{2 \mathrm{e}^{\beta \omega}\left\langle S_{z}\right\rangle}{\mathrm{e}^{\beta \omega}-1},
\end{aligned}
$$

where the second step is reached with properties of trace and the factor $\mathrm{e}^{-\beta \omega}$ comes from commuting $S_{+}$with $\mathrm{e}^{-\beta H_{m f}}$. Hence, (13) is reduced to

$$
\frac{4 \pi\left\langle S_{z}\right\rangle}{N \hbar} \sum_{\boldsymbol{q}} \delta\left(\varepsilon_{\boldsymbol{k},-}-\varepsilon_{\boldsymbol{k}-\boldsymbol{q},+}-\omega_{\boldsymbol{q}}\right)\left(b_{\boldsymbol{q}}+1\right)\left|M_{\boldsymbol{k}, \boldsymbol{k}-\boldsymbol{q},-}\right|^{2},
$$

where

$$
b_{\boldsymbol{q}}=\frac{1}{\mathrm{e}^{\beta \omega_{q}}-1}
$$

is the Bose-Einstein distribution function of magnons. Similarly, that of a hole in the state $\boldsymbol{k}, \sigma=1$ is

$$
\frac{4 \pi\left\langle S_{z}\right\rangle}{N \hbar} \sum_{\boldsymbol{p}} \delta\left(\varepsilon_{\boldsymbol{k},+}-\varepsilon_{\boldsymbol{k}+\boldsymbol{p},-}+\omega_{\boldsymbol{p}}\right) b_{\boldsymbol{p}}\left|M_{\boldsymbol{k}, \boldsymbol{k}+\boldsymbol{p},+}\right|^{2} .
$$

The scattering rate of a hole in the state $\boldsymbol{k}, \sigma=-1$ in a spin-conserving process is given by the first order in $H_{f l 2}$ and second order in $H_{f l 1}$ :

$$
\begin{gathered}
\frac{8 \pi\left\langle S_{z}\right\rangle^{2}}{N^{2} \hbar} \sum_{\boldsymbol{p}, \boldsymbol{q}} \delta\left(\varepsilon_{\boldsymbol{k},-}-\varepsilon_{\boldsymbol{k}-\boldsymbol{q}+\boldsymbol{p},-}-\omega_{\boldsymbol{q}}+\omega_{\boldsymbol{p}}\right) b_{\boldsymbol{p}}\left(b_{\boldsymbol{q}}+1\right) \\
\left|\varepsilon_{\boldsymbol{k}-\boldsymbol{q}}+\frac{\left[1-f_{0}\left(\varepsilon_{\boldsymbol{k}-\boldsymbol{q},+}\right)\right] M_{\boldsymbol{k}, \boldsymbol{k}-\boldsymbol{q},-} M_{\boldsymbol{k}-\boldsymbol{q}, \boldsymbol{k}-\boldsymbol{q}+\boldsymbol{p},+}}{\varepsilon_{\boldsymbol{k},-}-\varepsilon_{\boldsymbol{k}-\boldsymbol{q},+}-\omega_{\boldsymbol{q}}}\right|^{2},
\end{gathered}
$$

and that of a hole in the state $\boldsymbol{k}, \sigma=1$ is

$$
\begin{gathered}
\frac{8 \pi\left\langle S_{z}\right\rangle^{2}}{N^{2} \hbar} \sum_{\boldsymbol{p}, \boldsymbol{q}} \delta\left(\varepsilon_{\boldsymbol{k},+}-\varepsilon_{\boldsymbol{k}-\boldsymbol{q}+\boldsymbol{p},+}-\omega_{\boldsymbol{q}}+\omega_{\boldsymbol{p}}\right) b_{\boldsymbol{p}}\left(b_{\boldsymbol{q}}+1\right) \\
\left|\varepsilon_{\boldsymbol{k}+\boldsymbol{p}}+\frac{\left[1-f_{0}\left(\varepsilon_{\boldsymbol{k}+\boldsymbol{p},-}\right)\right] M_{\boldsymbol{k}, \boldsymbol{k}+\boldsymbol{p},+} M_{\boldsymbol{k}+\boldsymbol{p}, \boldsymbol{k}-\boldsymbol{q}+\boldsymbol{p},-}}{\varepsilon_{\boldsymbol{k},+}-\varepsilon_{\boldsymbol{k}+\boldsymbol{p},-}+\omega_{\boldsymbol{p}}}\right|^{2} .
\end{gathered}
$$

We have the Boltzmann equation for the holes of spin $\sigma:$

$$
e \boldsymbol{E} \cdot \frac{\hbar \boldsymbol{k}}{m_{\sigma}} \frac{\partial f_{0}\left(\varepsilon_{\boldsymbol{k}, \boldsymbol{\sigma}}\right)}{\partial \varepsilon_{\boldsymbol{k}}}=\left.\frac{\partial f_{\boldsymbol{k}, \boldsymbol{\sigma}}}{\partial t}\right|_{\text {sc.coll. }}+\left.\frac{\partial f_{\boldsymbol{k}, \boldsymbol{\sigma}}}{\partial t}\right|_{\text {sf.coll. }},
$$

where $f_{\boldsymbol{k} \boldsymbol{\sigma}}$ and $f_{0}\left(\varepsilon_{\boldsymbol{k} \boldsymbol{\sigma}}\right)$ are respectively, the nonequilibrium and Fermi-Dirac distribution functions of the holes with spin $\sigma$ and energy $\varepsilon_{\boldsymbol{k} \boldsymbol{\sigma}}$, and $m_{\sigma}$ is the effective mass of the holes. For the spin-flip scattering:

$$
\begin{aligned}
\left.\frac{\partial f_{\boldsymbol{k}-}}{\partial t}\right|_{s f . c o l l .}= & \frac{4 \pi\left\langle S_{z}\right\rangle}{N \hbar} \sum_{\boldsymbol{q}}\left[\left(1-f_{\boldsymbol{k},-}\right) f_{\boldsymbol{k}-\boldsymbol{q},+} b_{\boldsymbol{q}}-f_{\boldsymbol{k},-}\left(1-f_{\boldsymbol{k}-\boldsymbol{q},+}\right)\left(b_{\boldsymbol{q}}+1\right)\right] \\
& \times \delta\left(\varepsilon_{\boldsymbol{k},-}-\varepsilon_{\boldsymbol{k}-\boldsymbol{q},+}-\omega_{\boldsymbol{q}}\right)\left|M_{\boldsymbol{k}, \boldsymbol{k}-\boldsymbol{q},-}\right|^{2}, \\
\left.\frac{\partial f_{\boldsymbol{k}+}}{\partial t}\right|_{s f . c o l l .}= & \frac{4 \pi\left\langle S_{z}\right\rangle}{N \hbar} \sum_{\boldsymbol{p}}\left[\left(1-f_{\boldsymbol{k},+}\right) f_{\boldsymbol{k}+\boldsymbol{p},-}\left(b_{\boldsymbol{p}}+1\right)-f_{\boldsymbol{k},+}\left(1-f_{\boldsymbol{k}+\boldsymbol{p},-}\right) b_{\boldsymbol{p}}\right] \\
& \times \delta\left(\varepsilon_{\boldsymbol{k},+}-\varepsilon_{\boldsymbol{k}+\boldsymbol{p},-}+\omega_{\boldsymbol{p}}\right)\left|M_{\boldsymbol{k}, \boldsymbol{k}+\boldsymbol{p},+}\right|^{2},
\end{aligned}
$$

For the spin-conserving scattering

$$
\begin{aligned}
\left.\frac{\partial f_{\boldsymbol{k}-}}{\partial t}\right|_{\text {sc.coll. }}= & \frac{8 \pi\left\langle S_{z}\right\rangle^{2}}{N^{2} \hbar} \sum_{\boldsymbol{q}, \boldsymbol{p}}\left[b_{\boldsymbol{q}}\left(b_{\boldsymbol{p}}+1\right)\left(1-f_{\boldsymbol{k},-}\right) f_{\boldsymbol{k}-\boldsymbol{q}+\boldsymbol{p},-}-b_{\boldsymbol{p}}\left(b_{\boldsymbol{q}}+1\right)\right. \\
& \left.\times f_{\boldsymbol{k},-}\left(1-f_{\boldsymbol{k}-\boldsymbol{q}+\boldsymbol{p},-}\right)\right] \delta\left(\varepsilon_{\boldsymbol{k},-}-\varepsilon_{\boldsymbol{k}-\boldsymbol{q}+\boldsymbol{p},-}-\omega_{\boldsymbol{q}+} \omega_{\boldsymbol{p}}\right) \\
& \times\left|\varepsilon_{\boldsymbol{k}-\boldsymbol{q}}+\frac{\left[1-f_{0}\left(\varepsilon_{\boldsymbol{k}-\boldsymbol{q},+}\right)\right] M_{\boldsymbol{k}, \boldsymbol{k}-\boldsymbol{q},-} M_{\boldsymbol{k}-\boldsymbol{q}, \boldsymbol{k}-\boldsymbol{q}+\boldsymbol{p},+}}{\varepsilon_{\boldsymbol{k},-}-\varepsilon_{\boldsymbol{k}-\boldsymbol{q},+}-\omega_{\boldsymbol{q}}}\right|^{2},
\end{aligned}
$$


and

$$
\begin{aligned}
\left.\frac{\partial f_{\boldsymbol{k}+}}{\partial t}\right|_{\text {sc.coll. }}= & \frac{8 \pi\left\langle S_{z}\right\rangle^{2}}{N^{2} \hbar} \sum_{\boldsymbol{q}, \boldsymbol{p}}\left[b_{\boldsymbol{q}}\left(b_{\boldsymbol{p}}+1\right)\left(1-f_{\boldsymbol{k},+}\right) f_{\boldsymbol{k}-\boldsymbol{q}+\boldsymbol{p},+}-b_{\boldsymbol{p}}\left(b_{\boldsymbol{q}}+1\right)\right. \\
& \left.\times f_{\boldsymbol{k},+}\left(1-f_{\boldsymbol{k}-\boldsymbol{q}+\boldsymbol{p},+}\right)\right] \delta\left(\varepsilon_{\boldsymbol{k},+}-\varepsilon_{\boldsymbol{k}-\boldsymbol{q}+\boldsymbol{p},+}-\omega_{\boldsymbol{q}}+\omega_{\boldsymbol{p}}\right) \\
& \left|\varepsilon_{\boldsymbol{k}+\boldsymbol{p}}+\frac{\left[1-f_{0}\left(\varepsilon_{\boldsymbol{k}+\boldsymbol{p},-}\right)\right] M_{\boldsymbol{k}, \boldsymbol{k}+\boldsymbol{p},+} M_{\boldsymbol{k}+\boldsymbol{p}, \boldsymbol{k}-\boldsymbol{q}+\boldsymbol{p}-}}{\varepsilon_{\boldsymbol{k},+}-\varepsilon_{\boldsymbol{k}+\boldsymbol{p},-}+\omega_{\boldsymbol{p}}}\right|^{2} .
\end{aligned}
$$

We used the following ansaz to solve the Boltzmann equations:

$$
f_{\boldsymbol{k}, \sigma}=f_{0}\left(\varepsilon_{\boldsymbol{k}, \sigma}\right)+\phi_{\sigma} e \boldsymbol{E} \cdot \frac{\hbar \boldsymbol{k}}{m_{\sigma}} \frac{\partial f_{0}\left(\varepsilon_{\boldsymbol{k} \sigma}\right)}{\partial \varepsilon_{\boldsymbol{k} \sigma}},
$$

where $\phi_{\sigma}$ depends on the chemical potential only. Equation (26) in effect, assumed that the non-equilibrium part is centered around Fermi surface and its magnitude is proportional to the applied electric field. Substituting eq. (26) into eqs. (22)-(25), we get

$$
\begin{aligned}
\left.\frac{\partial f_{\boldsymbol{k}-}}{\partial t}\right|_{\text {sf.coll. }}= & \frac{4 \pi\left\langle S_{z}\right\rangle}{N \hbar k T} \sum_{\boldsymbol{q}}\left[1-f_{0}\left(\varepsilon_{\boldsymbol{k}-}\right)\right] f_{0}\left(\varepsilon_{\boldsymbol{k}-\boldsymbol{q},+}\right) b_{\boldsymbol{q}} \delta\left(\varepsilon_{\boldsymbol{k},-}-\varepsilon_{\boldsymbol{k}-\boldsymbol{q},+}-\omega_{\boldsymbol{q}}\right) \\
& \times e \boldsymbol{E} \cdot\left[\frac{\hbar \boldsymbol{k} \phi_{-}}{m_{-}}-\frac{\hbar(\boldsymbol{k}-\boldsymbol{q}) \phi_{+}}{m_{+}}\right]\left|M_{\boldsymbol{k}, \boldsymbol{k}-\boldsymbol{q},-}\right|^{2}, \\
\left.\frac{\partial f_{\boldsymbol{k}+}}{\partial t}\right|_{s f . c o l l .}= & \frac{4 \pi\left\langle S_{z}\right\rangle}{N \hbar k T} \sum_{\boldsymbol{p}}\left[1-f_{0}\left(\varepsilon_{\boldsymbol{k}+\boldsymbol{p},-}\right)\right] f_{0}\left(\varepsilon_{\boldsymbol{k},+}\right) b_{\boldsymbol{p}} \delta\left(\varepsilon_{\boldsymbol{k},+}-\varepsilon_{\boldsymbol{k}+\boldsymbol{p},-}+\omega_{\boldsymbol{p}}\right) \\
& \times e \boldsymbol{E} \cdot\left[\frac{\hbar \boldsymbol{k} \phi_{+}}{m_{+}}-\frac{\hbar(\boldsymbol{k}+\boldsymbol{p}) \phi_{-}}{m_{-}}\right]\left|M_{\boldsymbol{k}, \boldsymbol{k}+\boldsymbol{p},+}\right|^{2}, \\
\left.\frac{\partial f_{\boldsymbol{k}-}}{\partial t}\right|_{s c . \text { coll. }}= & \frac{8 \pi\left\langle S_{z}\right\rangle^{2}}{N^{2} \hbar k T} \sum_{\boldsymbol{q}, \boldsymbol{p}} f_{0}\left(\varepsilon_{\boldsymbol{k}-}\right)\left[1-f_{0}\left(\varepsilon_{\boldsymbol{k}-\boldsymbol{q}+\boldsymbol{p}-}\right)\right] b_{\boldsymbol{p}}\left(b_{\boldsymbol{q}}+1\right) \\
& \times \delta\left(\varepsilon_{\boldsymbol{k},-}-\varepsilon_{\boldsymbol{k}-\boldsymbol{q}+\boldsymbol{p},-}-\omega_{\boldsymbol{q}}+\omega_{\boldsymbol{p}}\right) e \boldsymbol{E} \cdot \frac{\hbar(\boldsymbol{q}-\boldsymbol{p}) \phi_{-}}{m_{-}} \\
& \times \mid \varepsilon_{\boldsymbol{k}-\boldsymbol{q}}+\frac{\left[1-f_{0}\left(\varepsilon_{\boldsymbol{k}-\boldsymbol{q}+}\right)\right] M_{\boldsymbol{k}, \boldsymbol{k}-\boldsymbol{q},-} M_{\boldsymbol{k}-\boldsymbol{q}, \boldsymbol{k}-\boldsymbol{q}+\boldsymbol{p},+}}{\varepsilon_{\boldsymbol{k},-}-\varepsilon_{\boldsymbol{k}-\boldsymbol{q}+}-\omega_{\boldsymbol{q}}},
\end{aligned}
$$

and

$$
\begin{aligned}
\left.\frac{\partial f_{\boldsymbol{k}+}}{\partial t}\right|_{\text {coll. }}= & \frac{8 \pi\left\langle S_{z}\right\rangle^{2}}{N^{2} \hbar k T} \sum_{\boldsymbol{q}, \boldsymbol{p}} f_{0}\left(\varepsilon_{\boldsymbol{k}+}\right)\left[1-f_{0}\left(\varepsilon_{\boldsymbol{k}-\boldsymbol{q}+\boldsymbol{p}+}\right)\right] b_{\boldsymbol{p}}\left(b_{\boldsymbol{q}}+1\right) \\
& \times \delta\left(\varepsilon_{\boldsymbol{k},+}-\varepsilon_{\boldsymbol{k}-\boldsymbol{q}+\boldsymbol{p},+}-\omega_{\boldsymbol{q}}+\omega_{\boldsymbol{p}}\right) e \boldsymbol{E} \cdot \frac{\hbar(\boldsymbol{q}-\boldsymbol{p}) \phi_{+}}{m_{+}} \\
& \times\left|\varepsilon_{\boldsymbol{k}+\boldsymbol{p}}+\frac{\left[1-f_{0}\left(\varepsilon_{\boldsymbol{k}+\boldsymbol{p}-}\right)\right] M_{\boldsymbol{k}, \boldsymbol{k}+\boldsymbol{p},+} M_{\boldsymbol{k}+\boldsymbol{p}, \boldsymbol{k}-\boldsymbol{q}+\boldsymbol{p},-}}{\varepsilon_{\boldsymbol{k}+}-\varepsilon_{\boldsymbol{k}+\boldsymbol{p}-}+\omega_{\boldsymbol{p}}}\right|^{2} .
\end{aligned}
$$

Taking the advantage of the fact that $\phi_{\sigma}$ is independent of $\boldsymbol{k}$, we substituted eqs. (27)-(30) into eq. (21), multiplied both sides by $e \boldsymbol{E} \cdot \hbar \boldsymbol{k} / \boldsymbol{m}_{\sigma}$, and summed over $\boldsymbol{k}$, to obtain

$$
v_{-}=y_{-} \phi_{-}+w_{-} \phi_{-}-w_{0} \phi_{+},
$$

and

$$
v_{+}=y_{+} \phi_{+}+w_{+} \phi_{+}-w_{0} \phi_{-},
$$

where

$$
\begin{aligned}
v_{\sigma} & =\frac{1}{N} \sum_{\boldsymbol{k}}\left(\frac{\hbar \boldsymbol{k}}{m_{\sigma}}\right)^{2} \frac{\partial f_{0}\left(\varepsilon_{\boldsymbol{k} \boldsymbol{\sigma}}\right)}{\partial \varepsilon_{\boldsymbol{k} \boldsymbol{\sigma}}}, \\
w_{-} & =-\frac{4 \pi\left\langle S_{z}\right\rangle}{N^{2} \hbar k T} \sum_{\boldsymbol{k}, \boldsymbol{q}}\left[1-f_{0}\left(\varepsilon_{\boldsymbol{k},-}\right)\right] f_{0}\left(\varepsilon_{\boldsymbol{k}-\boldsymbol{q},+}\right) b_{\boldsymbol{q}} \delta\left(\varepsilon_{\boldsymbol{k},-}-\varepsilon_{\boldsymbol{k}-\boldsymbol{q},+}-\omega_{\boldsymbol{q}}\right)\left(\frac{\hbar \boldsymbol{k}}{m_{-}}\right)^{2}\left|M_{\boldsymbol{k}, \boldsymbol{k}-\boldsymbol{q},-}\right|^{2}, \\
w_{+} & =-\frac{4 \pi\left\langle S_{z}\right\rangle}{N^{2} \hbar k T} \sum_{\boldsymbol{k}, \boldsymbol{p}}\left[1-f_{0}\left(\varepsilon_{\boldsymbol{k},+}\right)\right] f_{0}\left(\varepsilon_{\boldsymbol{k}+\boldsymbol{p},-}\right)\left(b_{\boldsymbol{p}}+1\right) \delta\left(\varepsilon_{\boldsymbol{k},+}-\varepsilon_{\boldsymbol{k}+\boldsymbol{p},-}+\omega_{\boldsymbol{p}}\right)\left(\frac{\hbar \boldsymbol{k}}{m_{+}}\right)^{2}\left|M_{\boldsymbol{k}, \boldsymbol{k}+\boldsymbol{p},+}\right|^{2},
\end{aligned}
$$




$$
\begin{aligned}
w_{0}= & -\frac{4 \pi\left\langle S_{z}\right\rangle}{N^{2} \hbar k T} \sum_{\boldsymbol{k}, \boldsymbol{q}}\left[1-f_{0}\left(\varepsilon_{\boldsymbol{k},-}\right)\right] f_{0}\left(\varepsilon_{\boldsymbol{k}-\boldsymbol{q},+}\right) b_{\boldsymbol{q}} \delta\left(\varepsilon_{\boldsymbol{k},-}-\varepsilon_{\boldsymbol{k}-\boldsymbol{q},+}-\omega_{\boldsymbol{q}}\right) \frac{\hbar \boldsymbol{k}}{m_{-}} \cdot \frac{\hbar(\boldsymbol{k}-\boldsymbol{q})}{m_{+}}\left|M_{\boldsymbol{k}, \boldsymbol{k}-\boldsymbol{q},-}\right|^{2}, \\
y_{-}= & -\frac{8 \pi\left\langle S_{z}\right\rangle^{2}}{N^{3} \hbar k T} \sum_{\boldsymbol{k}, \boldsymbol{p}, \boldsymbol{q}} f_{0}\left(\varepsilon_{\boldsymbol{k}-}\right)\left[1-f_{0}\left(\varepsilon_{\boldsymbol{k}-\boldsymbol{q}+\boldsymbol{p}-}\right)\right] b_{\boldsymbol{p}}\left(b_{\boldsymbol{q}}+1\right) \\
& \times \delta\left(\varepsilon_{\boldsymbol{k},-}-\varepsilon_{\boldsymbol{k}-\boldsymbol{q}+\boldsymbol{p},-}-\omega_{\boldsymbol{q}}+\omega_{\boldsymbol{p}}\right) \frac{\hbar \boldsymbol{k}}{m_{-}} \cdot \frac{\hbar(\boldsymbol{q}-\boldsymbol{p})}{m_{-}} \\
& \times\left|\varepsilon_{\boldsymbol{k}-\boldsymbol{q}}+\frac{\left[1-f_{0}\left(\varepsilon_{\boldsymbol{k}-\boldsymbol{q},+}\right)\right] M_{\boldsymbol{k}, \boldsymbol{k}-\boldsymbol{q},-} M_{\boldsymbol{k}-\boldsymbol{q}, \boldsymbol{k}-\boldsymbol{q}+\boldsymbol{p},+}}{\varepsilon_{\boldsymbol{k}-}-\varepsilon_{\boldsymbol{k}-\boldsymbol{q}+}-\omega_{\boldsymbol{q}}}\right|^{2},
\end{aligned}
$$

and

$$
\begin{aligned}
y_{+}= & -\frac{8 \pi\left\langle S_{z}\right\rangle^{2}}{N^{3} \hbar k T} \sum_{\boldsymbol{k}, \boldsymbol{p}, \boldsymbol{q}} f_{0}\left(\varepsilon_{\boldsymbol{k},+}\right)\left[1-f_{0}\left(\varepsilon_{\boldsymbol{k}-\boldsymbol{q}+\boldsymbol{p},+}\right)\right] b_{\boldsymbol{p}}\left(b_{\boldsymbol{q}}+1\right) \\
& \times \delta\left(\varepsilon_{\boldsymbol{k},+}-\varepsilon_{\boldsymbol{k}-\boldsymbol{q}+\boldsymbol{p},+}-\omega_{\boldsymbol{q}}+\omega_{\boldsymbol{p}}\right) \frac{\hbar \boldsymbol{k}}{m_{+}} \cdot \frac{\hbar(\boldsymbol{q}-\boldsymbol{p})}{m_{+}} \\
& \times\left|\varepsilon_{\boldsymbol{k}+\boldsymbol{p}}+\frac{\left[1-f_{0}\left(\varepsilon_{\boldsymbol{k}+\boldsymbol{p}-}\right)\right] M_{\boldsymbol{k}, \boldsymbol{k}+\boldsymbol{p},+} M_{\boldsymbol{k}+\boldsymbol{p}, \boldsymbol{k}-\boldsymbol{q}+\boldsymbol{p},-}}{\varepsilon_{\boldsymbol{k}+}-\varepsilon_{\boldsymbol{k}+\boldsymbol{p}-}+\omega_{\boldsymbol{p}}}\right|^{2} .
\end{aligned}
$$

Having Calculated $\nu_{\sigma}, w_{\sigma}, w_{0}$ and $y_{\sigma}$, we can evaluate $\phi_{\sigma}$ and hence, the conductivity of spin \pm holes

$$
\sigma_{ \pm}=\frac{e^{2} \phi_{ \pm}}{3 V} \sum_{\boldsymbol{k}}\left(\frac{\hbar \boldsymbol{k}}{m_{ \pm}}\right)^{2} \frac{\partial f_{0}\left(\varepsilon_{\boldsymbol{k} \pm}\right)}{\partial \varepsilon_{\boldsymbol{k} \pm}}
$$

Finally, the resistivity is

$$
\rho=\frac{1}{\sigma_{+}+\sigma_{-}} .
$$

We will show our results in next section.

\section{§4. Results and Discussion}

The resulting resisitivities are shown in Fig. 1 where the solid, dotted and dashed lines denoting that at doping concentration $x=0.2,1 / 3$ and 0.45 respectively. Figure 1(b) is a log-log plot which shows that the behavior of resistivity is not a simple power law of temperature. In the double exchange systems, the temperature dependence of resistivity is quite complicated. We have accounted for three processes, namely the spin-flip, second order in one magnon and two magnons. The last one (from $H_{f l 2}$ ) is the dominating process. Only at very high temperature $\left(T \geq 0.8 T_{\mathrm{C}}\right)$ the spin-flip process has significant contribution. We also found that only in that temperature range the current of minority holes is significant. This can be understood from the band picture. As temperature rises the spin-up band and spin-down band move closer to each other. As a result, the minority spin holes become abundant and spin-flip becomes energetically more favorable. A recent study ${ }^{16)}$ on similar systems shows that the spin-flip (one-magnon scattering) process gives a resistivity proportional to $T^{3}$. But in a pure DE system with single band as we have here, two-magnon process has the most phase space available because of the requirement of energy-conservation.

In KO's calculation the resistivity is proportional to $T^{9 / 2}$. This is only valid in low temperature range where the average of local spin $\left\langle S_{z}\right\rangle$ is near its saturated value. As temperature increases, several effects should be con- sidered. The band structure of holes changes. The spinup band and spin-down band move toward each other and hence, scatterings occur more frequently. More importantly, the coupling constant depends upon $\left\langle S_{z}\right\rangle$ (eqs. (16), (18)-(20)) and thus upon temperature. The scattering becomes weaker with increasing temperature. Therefore KO's result is only applicable in temperature region, $T<T_{\mathrm{C}} / 4$, where the magnetization of the local spins is near its saturation value (see Fig. 2). To sum-
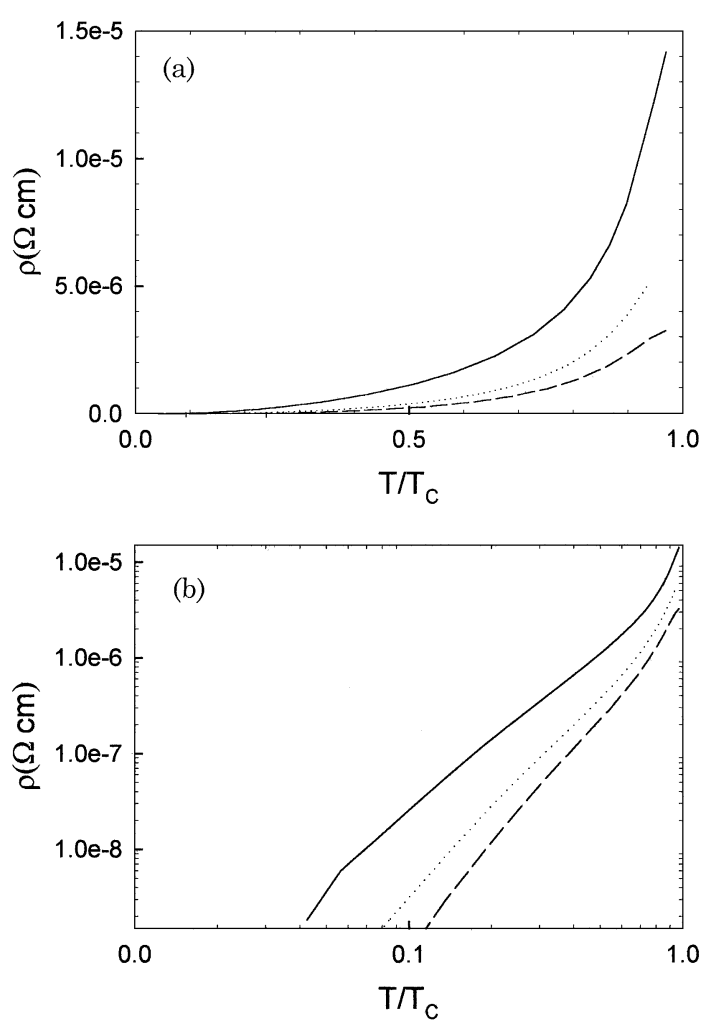

Fig. 1. Resistivity versus temperature. The results at doping $x=$ $0.2,1 / 3$ and 0.45 are represented by the solid, dotted and dashed lines respectively. Figure $1(\mathrm{~b})$ is a log-log plot. 


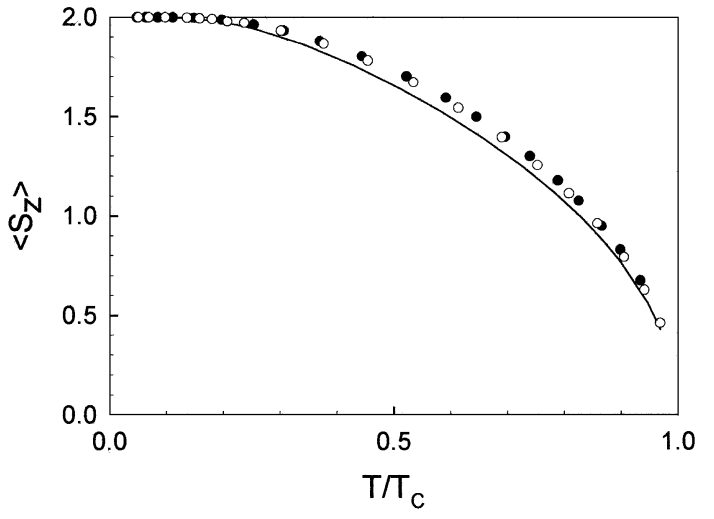

Fig. 2. Average local spin $\left\langle S_{z}\right\rangle$ versus temperature. The results at doping $x=0.2,1 / 3$ and 0.45 are represented by the solid line, dots and open circles respectively.

marize the behavior the resistivity, we stated that it is closely related to magnetization.

The comparison with experiments is somewhat disappointing. In ref. 11 the measured resistivity is proportional to $T^{2}$. More importantly, the magnitude of resistivity is much greater than that from our calculation, In fact, all the calculations using double exchange models ${ }^{17)}$ have this difficulty. Therefore, it is clear that the holespin wave scattering cannot give such a large resistivity found by experiments. One possible candidate for such large resistivity is the electron-electron interaction. According to Ziman, ${ }^{18}$ ) the resitivity due to e-e scattering has the form

$$
\rho_{e e} \sim \frac{\pi^{3} z^{\prime} \mathcal{G}^{2} a}{64 v_{F}}\left(\frac{k T}{E_{F}}\right)^{2}
$$

where $a$ is the lattice constant, $v_{F}$ and $E_{F}$ are the Fermi velocity and energy, $z^{\prime}$ is the coordination in reciprocal lattice and $\mathcal{G}$ is the interference factor of the wave functions. Substituting the values for $\mathrm{La}_{1-x} \mathrm{Ga}_{x} \mathrm{MnO}_{3}$, $a \sim 6 \times 10^{-8} \mathrm{~cm}, v_{F} \sim 7 \times 10^{7} \mathrm{~cm} / \mathrm{s}, E_{F} \sim 0.7 \mathrm{eV}$, we found

$$
\rho_{e e} \sim 10^{-2} \mathcal{G}^{2}\left(\frac{k T}{E_{F}}\right)^{2} \Omega \mathrm{cm},
$$

which is still too small if we set $\mathcal{G}$ to be 1 . Another possi- bility is that there may be spin fluctuation unaccounted for. In a previous work we calculated the Cutie temperature using $H_{m f}$ in eq. (5). The resulting $T_{\mathrm{C}}$ is about three times of the experimental value. Adding superexchange interaction (SE) with strenght about $t / 30$ will bring $T_{\mathrm{C}}$ to realistic values. The presence of $\mathrm{SE}$ which favors antiferromagnetism can enhance spin fluctuation and change the behavior of resistivity. The third hopeful mechanism is the polaron effect. ${ }^{19)}$ We think it is still not clear at this stage.

In conclusion, we have calculated the resistivity in the double exchange model. What we found is that in this model the spin wave-hole scattering is too small to give a resistivity compatible with experimental results.

This work was supported in part by the National Science Council of Taiwan, ROC, under the contract NSC 89-2112-M-002-026.

1) G. H. Jonker and J. H. Santen: Physics 16 (1950) 337.

2) E. O. Wollen and W. C. Koehler: Phys. Rev. 100 (1955) 548.

3) C. Zener: Phys. Rev. 82 (1951) 403.

4) P. W. Anderson and Hasegawa: Phys. Rev. 100 (1955) 675.

5) P. G. de Gennes: Phys. Rev. 118 (1960) 141.

6) K. Kubo and N. Ohata: J. Phys. Soc. Jpn. 33 (1972) 21.

7) Nobuo Furukawa: J. Phys. Soc. Jpn. 63 (1994) 3214.

8) L. Sheng, H. Y. Tong, D. N. Sheng and C. S. Ting: Phys. Rev. B 58 (1997) 8186.

9) A. J. Millis, P. B. Littlewood and B. I. Shraiman: Phys. Rev. Lett. 74 (1995) 5144; J. Millis, Boris I. Shraiman and R. Mueller: Phys. Rev. Lett. 77 (1996) 175.

10) See for example S. Mori, C. H. Chen and S.-M. Cheong: Nature 392 (1998) 473.

11) See for example, P. Schiffer, A. P. Ramirez, W. Bao and S-W. Cheong: Phys. Rev. Lett. 75 (1995) 3336.

12) W. E. Pickett and D. J. Singh: Phys. Rev. B 53 (1996) 1146; D. J. Singh and W. E. Pickett: Phys. Rev. B 57 (1998) 88.

13) C. D. Hu: J. Phys. Soc. Jpn. 69 (2000) 3704.

14) C. D. Hu: J. Phys. Soc. Jpn. 69 (2000) 3411.

15) J. A. Fernandez-Baca, P. Dai, H. Y. Hwang, C. Kloc and S.-W. Cheong: Phys. Rev. Lett. 80 (1998) 4012; J. W. Lynn, R. W. Erwin, J. A. Borchers, Q. Huang and A. Santoro: Phys. Rev. Lett. 76 (1996) 4046.

16) Nobuo Furukawa: J. Phys. Soc. Jpn. 69 (2000) 1954.

17) See for example, D. M. Edwards, A. C. M. Green and K. Kubo: J. Phys.: Condens. Matter 11 (1999) 2791.

18) J. M. Ziman: Electrons and Phonons (Oxford, 1960).

19) See for example, H. Röder, Jun Zang and A. R. Bishop: Phys. Rev. Lett. 76 (1996) 1356. 